DOI: 10.12731/2658-6649-2020-12-2-93-105

УДК 616.72-002.772

\title{
ЛЕЧЕНИЕ ПАЦИЕНТОВ С РЕВМАТОИДНЫМ АРТРИТОМ ПРИ СОЧЕТАННОЙ ПАТОЛОГИИ
}

\author{
Хусаинова Д.И., Бакиров Б.А., Зарипова Г.Р., Хусаинова Л.Н., \\ Аитова Э.М., Баянова И.Л., Музаева И.Л., Хакамова Г.А.
}

Сочетанная патология у пациентов с ревматоидным артритом. Bblявление взаимосвязи с эффектом от медикаментозной терапии.

Ключевые слова: ревматоидный артрит; коморбидная патология; генно-инженерные биологические препараты.

\section{TREATMENT OF PATIENTS WITH RHEUMATOID ARTHRITIS WITH COMBINED PATHOLOGY}

\author{
Khusainova D.I., Bakirov B.A., Zaripova G.R., Khusainova L.N., \\ Aitova E.M., Bayanova I.L., Muzaeva I.L., Khakamova G.A.
}

Combined pathology in patients with rheumatoid arthritis. Identification of the relationship with the effect of drug therapy.

Keywords: rheumatoid arthritis; comorbid pathology; genetic engineering biological preparations.

\section{Введение}

Распространенность РА составляет 0,5-2\% [5]. По данным Стародубцевой И.А. и соавторов (2016), частота встречаемости вторичного остеортроза у пациентов с Р составляет 100\%. Помимо патологии ОДА у пациентов также превалировали артериальная гипертензия (38,3\%), ИБС (10,1\%), заболевания ЖКТ (55\%). У больных РА диагностировано 2 и более коморбидных заболевания $(58,6 \%)$ [9].

Имеется прямая взаимосвязь между активностью воспаления при РА и риском СС3. Так, например, пациенты с высокими показателями активности по основному заболеванию характеризовались более выраженной скоростью прогрессирования атеросклероза. Данная взаимосвязь с патогенетической точки зрения может быть обусловлена изменением структуры 
проатерогенных липопротеинов вследствие хронического воспаления, а также уменьшением синтеза и биодоступности NO.

В дебюте ИБС, в свою очередь, имеет значение индукция факторов адгезии и агрегации тромбоцитов, экспрессия тканевого фактора, индуцированная воспалительными цитокинами. Клиническая манифестация ИБС редко проявляет себя в классическом болевом варианте. Чаще всего дебютом ИБС на фоне РА является безболевая ишемия миокарда либо уже развившиеся более серьезные осложнения $[8,16]$.

Риск ишемического инсульта у пациентов с РА в среднем на $30 \%$ певышает таковой у основной популяции. Независимым фактором риска при этом выступает артериальная гипертензия как в роли сопутствующей патологии, так и в качестве макрососудистого осложнения РА.

По данным Панафидиной ТА и соавторов кардиоваскулярная патология встречается у 22\% пациентов и преимущественно представлена ИБС. Частота АГ варьирует в диапазоне 18-70\%, а частота сахарного диабета порядка 15-19\%. Данное явление, по мнению автора, может быть связано с более высокой частотой манифестации атеросклероза на фоне хронического воспаления, побочных эффектов противоревматической терапии и недостаточным охватом профилактикой СС3. При этом около 81\% коморбидных по АГ пациентов получают регулярную гипотензивную терапию. При этом частота приема статинов не достигает 30\%, а дезагрегантов с профилактической целью - не более 9,5\%.

У пациентов с РА за несколько лет до манифестации основного заболевания обнаруживаются предикторы атеросклеротических процессов в виде повышения уровня атерогенных фракций липидов [7, 20].

Цель настоящего исследования - изучить частоту встречаемости коморбидной патологии при РА.

\section{Объект и методы исследования}

С целью выявления характера коморбидной патологии у пациентов с РА и ее взаимосвязей с эффектом от медикаментозной терапии РА в условиях специализированного стационара нами проанализирована медицинская документация 163 пациента, госпитализированных в терапевтическое отделение Клиники ФГБОУ ВО БГМУ за период с 2015 года по 2018 гг.

Для верификации диагноза использовались Классификационные критерии ревматоидного артрита ACR/EULAR 2010 г. (American College of Rheumatology/European League Against Rheumatism Rheumatoid arthritis classification criteria). Для определения рентгенологической стадии ис- 
пользовали модифицированную классификацию РА по Штейнброкеру. Для оценки оценки активности PA использовали индекс DAS28 - счет активности болезни (Disease Activity Score) для 28 суставов (в модификациях с применением СОЭ и СРБ) по формуле для вычисления DAS28: $\mathrm{DAS} 28=0,56 \sqrt{Ч Б C}+0,28 \sqrt{ }$ ЧПС $+0,70 \operatorname{lnCOЭ}+0,0140 О З Б$. Оценка функционального класса осложнений проводилась в соответствии с Клиническими рекомендациями по диагностике и лечению ревматоидного артрита Общероссийской общественной организации «Ассоциация ревматологов России» (2013 г). Титр РФ определяли методом латекс-агглютинации, титр анти-ЦЦП-методом иммуноферментного анализа.

У всех обследуемых при поступлении в Клинику и в некоторых случаях в динамике, помимо общепринятого клинико-лабораторного обследования (ЭКГ, ОАК, ОАМ), определяли биохимические показатели (АсАТ, АлАТ, общий белок, липидный профиль, мочевина, креатинин, мочевая кислота, щелочная фосфатаза, глюкоза крови, РФ, АЦЦП).

\section{Результаты исследования}

Клинико-демографическая характеристика госпитализированных пациентов представлена в таблицах 1 и 2.

Таблицุа 1.

Демографические показатели исследуемых пациентов

\begin{tabular}{|l|l|}
\hline \multicolumn{1}{|c|}{ Признак } & \multicolumn{1}{|c|}{ Показатель } \\
\hline Средний возраст, лет & 54,63 \\
\hline Мужчины (\%) & $36(22,09 \%)$ \\
\hline Женщины (\%) & $127(77,91 \%)$ \\
\hline Средняя продолжительность заболевания, лет & 9,56 \\
\hline
\end{tabular}

Таблийа 2.

Клинические характеристики пациентов

\begin{tabular}{|l|l|l|}
\hline \multicolumn{1}{|c|}{ Признак } & \multicolumn{1}{|c|}{ Стадия } & \multicolumn{1}{|c|}{$\begin{array}{c}\text { Частота } \\
\text { встречаемости }\end{array}$} \\
\hline Стадия заболевания & Очень ранняя стадия & 0 \\
\hline & Ранняя стадия & $49(30,3 \%)$ \\
\hline & Развернутая стадия & $74(45,7 \%)$ \\
\hline & Поздняя стадия & $40(24 \%)$ \\
\hline Форма заболевания & $\begin{array}{l}\text { Суставная (без системных } \\
\text { проявлений) }\end{array}$ & $117(71,78 \%)$ \\
\hline & Системные проявления & $46(28,22 \%)$ \\
\hline
\end{tabular}


Окончание табл. 2.

\begin{tabular}{|l|l|l|}
\hline Серо-принадлежность & Серопозитивный & $147(90,18 \%)$ \\
\hline & Серонегативный & $16(9,82 \%)$ \\
\hline & Не определена & 0 \\
\hline & І & $6(3,68 \%)$ \\
\hline & II & $81(49,69 \%)$ \\
\hline & III & $69(42,34 \%)$ \\
\hline $\begin{array}{l}\text { Активнен-стадия } \\
\text { заболевания }\end{array}$ & IV & $7(4,29 \%)$ \\
\hline & 1 & $3(1,84 \%)$ \\
\hline & 2 & $78(47,85 \%)$ \\
\hline $\begin{array}{l}\text { Индекс DAS (среднее } \\
\text { значение) }\end{array}$ & 3 & $82(50,31 \%)$ \\
\hline $\begin{array}{l}\text { Иммунологическая } \\
\text { характеристика }\end{array}$ & АЦЦП-позитивный & \\
\hline & АЦЦП- негативный & $143(87,73 \%)$ \\
\hline & АЦЦП не опред & $12(7,54 \%)$ \\
\hline $\begin{array}{l}\text { Функциональный } \\
\text { класс (НФС) } \\
\text { осложнений }\end{array}$ & 0 & $8(4,73 \%)$ \\
\hline & I & $3(1,84 \%)$ \\
\hline & II & $8(4,9 \%)$ \\
\hline & III & $128(78,53 \%)$ \\
\hline
\end{tabular}

Средний возраст пациентов составил 54,63 года. Продолжительность заболевания варьировала от 3 месяцев до 30 лет (среднее значение - 9,56 лет). Среди госпитализированных пациентов преобладали больные с суставной формой РА $(71,78 \% \%)$, преимущественно в развернутой стадии заболевания (45,7 \%). Среди пациентов с установленным диагнозом РА преобладали представители женского пола $(77,91 \%)$.

Серопозитивный РА диагностирован у $148(90,18)$ пациента, серонегативный - 12 (9,22\%) пациентов . Активность заболевания расценивалась как 3 у пациентов с РА более, чем в половине случаев (50,31\%).

Положительный результат по АЦЦП установлен у 143 (87,73\%) пациентов.

Нарушение функции суставов соответствовало в большинстве случаев II функциональному классу - 128 (78,53\%) Среди осложнений основного заболевания превалировал вторичный остеоартроз ( 41,49\%), а также вторичный остеопороз (16\%) и контрактура суставов $(12,77 \%)$. 
У 46 (28,22\%) пациентов определяли внесуставные проявления РА. Структура осложнений ревматоидного артрита представлена в таблице 3.

Таблица 3.

Частота осложнений ревматоидного артрита

\begin{tabular}{|l|c|}
\hline \multicolumn{1}{|c|}{ Признак } & Частота выявлений \\
\hline Остеоартроз вторичный & 39 \\
\hline Асептический некроз головки бедра & 7 \\
\hline Гормонозависимость & 18 \\
\hline Контрактура суставов & 12 \\
\hline Остеопороз & 15 \\
\hline Патологический перелом & 3 \\
\hline Протезирование сустава & 5 \\
\hline Сакроилеит & 1 \\
\hline Ревматические узелки & 21 \\
\hline Лихорадка & 15 \\
\hline Потеря массы тела & 5 \\
\hline Кардит, перикардит & 3 \\
\hline Нейропатия & 3 \\
\hline Ксеростомия & 2 \\
\hline Нефрит & 2 \\
\hline Пневмонит & 1 \\
\hline Увеит & 1 \\
\hline Васкулит & 1 \\
\hline Пневмофиброз & 1 \\
\hline
\end{tabular}

По результатам исследования наличие сопутствующей патологии выявлено у 103 пациентов (63,2\%). Количество сопутствующей патологии коррелировало с возрастом пациентов, что представлено в таблице 4.

\section{Количество нозологий у пациента с ревматоидным артритом}

Таблица 4.

в зависимости от возраста

\begin{tabular}{|c|c|}
\hline Возраст & Число нозологий \\
\hline 35 & 1,5 \\
\hline 50 & 1,389 \\
\hline 60 & 1,957 \\
\hline 70 & 3,488 \\
\hline 85 & 3,0 \\
\hline
\end{tabular}


Коморбидная патология была представлена преимущественно заболеваниями сердечно-сосудистой системы (39,09\%) (рис 1) .

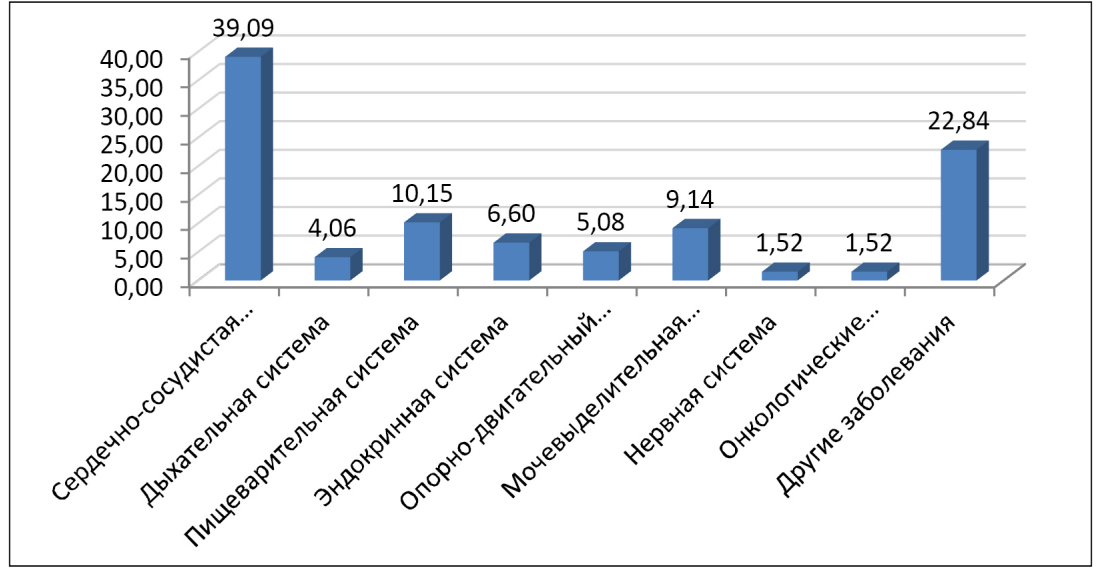

Рис. 1. Поражение различных систем у пациентов с ревматоидным артритом

Частота встречаемости сопутствующих заболеваний у пациентов с РА составила от 1 до 4 заболеваний у одного пациента.

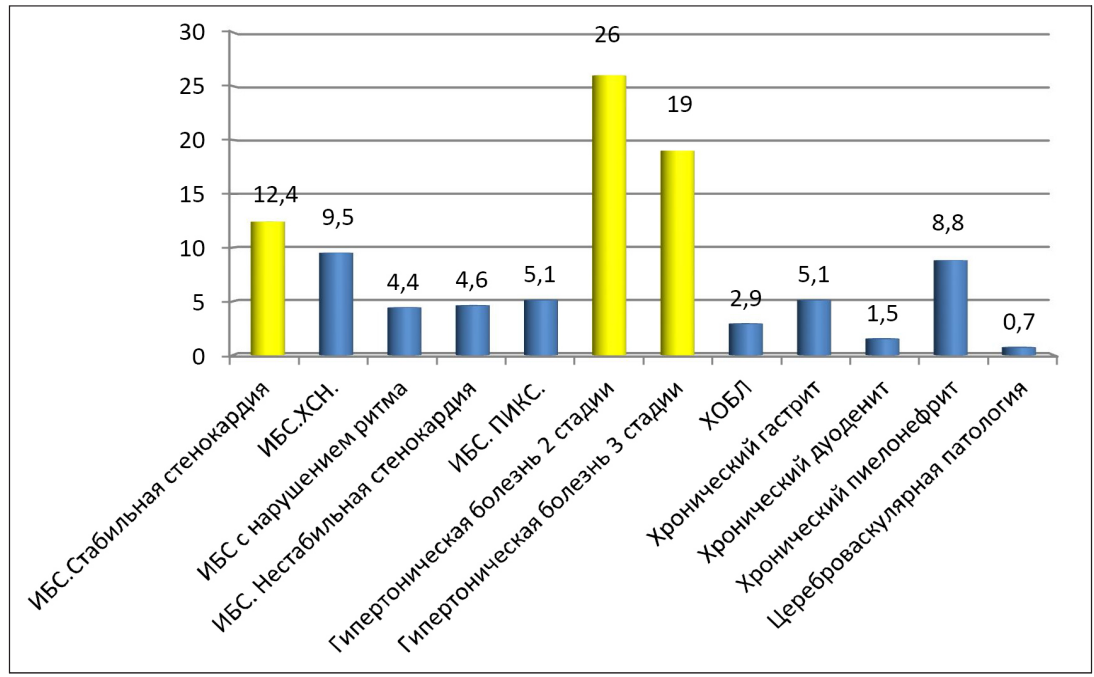

Рис. 2. Частота сопутствующей патологии у больных с ревматоидным артритом 
Среднее количество сопутствующих заболеваний на одного пациента с РА было представлено следующим образом: у 32 пациента диагностировано 1 сопутствующее заболевание, у 16 пациентов - 2 сопутствующих заболевания, у 9 пациентов - 3 сопутствующих заболевания, у 5 пациентов -4 сопутствующих заболевания. Количество нозологий у одного пациента было различным в зависимости от возраста. Наибольшим количеством сопутствующих заболеваний характеризовалась группа пациентов в возрасте $60-70$ лет $(1,9-3,4)$. Из сопутствующих заболеваний у пациентов с ревматоидным артритом наиболее часто сочетались артериальная гипертония, ишемическая болезнь сердца. Частота сопутствующей патологии представлена на рисунке 2 .

При сравнении группы с коморбидным состоянием и без у пациентов с ревматоидным артритом достоверных различий в клинической характеристике не выявлено, кроме увеличения количества назначенных препаратов.

Таблииза 5.

Клинико-демографическая характеристика пациентов в группах сравнения

\begin{tabular}{|c|c|c|c|c|c|c|}
\hline \multirow[t]{2}{*}{ Показатель } & \multicolumn{2}{|c|}{$\begin{array}{c}\text { Группа пациен- } \\
\text { тов с коморбид- } \\
\text { ными состояни- } \\
\text { ями (сопутству- } \\
\text { ющими) } \\
\end{array}$} & \multicolumn{2}{|c|}{$\begin{array}{c}\text { Группа па- } \\
\text { циентов без } \\
\text { коморбидных } \\
\text { состояний (со- } \\
\text { путствующих) }\end{array}$} & \multicolumn{2}{|c|}{ Вся выборка } \\
\hline & Чел. & \begin{tabular}{|l|}
$\%$ \\
\end{tabular} & Чел. & $\%$ & Чел. & $\%$ \\
\hline $\begin{array}{l}\text { Всего (абс, \% к общему числу } \\
\text { случаев) }\end{array}$ & 103 & 63,2 & 60 & 36,8 & 163 & 100 \\
\hline Женщины (абс, \% к группе) & 75 & 72,8 & 52 & 86,7 & 127 & 77,91 \\
\hline Мужчины (абс, \% к группе) & 28 & 27,18 & 8 & 13,33 & 36 & 22,09 \\
\hline Возраст (средний) & \multicolumn{2}{|c|}{59,12} & \multicolumn{2}{|c|}{46,95} & \multicolumn{2}{|c|}{54,64} \\
\hline Продолжительность РА & 9,5 & & 9,95 & & 9,67 & \\
\hline Серопозитивные по РФ & 93 & 90,3 & 54 & 90,0 & 147 & 90,18 \\
\hline Серопозитивные по АЦЦП & 87 & 84,5 & 56 & 93,33 & 143 & 87,73 \\
\hline DAS 28 (среднее значение) & \multicolumn{2}{|l|}{5,27} & \multicolumn{2}{|l|}{5,50} & \multicolumn{2}{|l|}{5,35} \\
\hline Наличие системных проявлений & 33 & 32,04 & 33 & 55,0 & 66 & 40,5 \\
\hline $\begin{array}{l}\text { Среднее количество назначен- } \\
\text { ных препаратов (предшествую- } \\
\text { щая терапия) }\end{array}$ & 4,94 & & 2,90 & & 3,93 & \\
\hline $\begin{array}{l}\text { Среднее количество назна- } \\
\text { ченных препаратов (основное } \\
\text { заболевание) }\end{array}$ & 5,09 & & 4,38 & & 4,6 & \\
\hline $\begin{array}{l}\text { Среднее количество назначен- } \\
\text { ных препаратов (БПВП) }\end{array}$ & 1,68 & & 2,03 & & 1,81 & \\
\hline
\end{tabular}


Однако по лабораторным и клиническим проявлениям, более выраженная симптоматика активности процесса была в группе пациентов с коморбидным фоном. Данные показатели представлены в таблице 6.

Таблицча 6.

Динамика клинико-лабораторных показателей в группах сравнения

\begin{tabular}{|c|c|c|c|c|c|c|}
\hline \multirow[t]{2}{*}{ Показатель } & \multicolumn{2}{|c|}{$\begin{array}{c}\text { Группа пациен- } \\
\text { тов с коморбид- } \\
\text { ными состояни- } \\
\text { ями (сопутству- } \\
\text { ющими) } \\
\end{array}$} & \multicolumn{2}{|c|}{$\begin{array}{c}\text { Группа па- } \\
\text { циентов без } \\
\text { коморбидных } \\
\text { состояний (со- } \\
\text { путствующих) }\end{array}$} & \multicolumn{2}{|c|}{ Вся выборка } \\
\hline & Чел. & $\%$ & Чел. & $\%$ & Чел. & $\%$ \\
\hline Без динамики & 5 & 5 & 1 & 0,02 & 6 & 3,7 \\
\hline Болевой синдром & 28 & 28 & 16 & 22,22 & 44 & 26,99 \\
\hline Динамика СОЭ & 37 & 37,0 & 14 & 22,22 & 51 & 31,29 \\
\hline Объём движения сустава & 44 & 44,0 & 31 & 49,21 & 75 & 46,01 \\
\hline Покраснение в области суставов & 44 & 44,0 & 29 & 46,03 & 73 & 44,79 \\
\hline Припухлость суставов & 69 & 69,0 & 49 & 77,78 & 118 & 72,39 \\
\hline Сила мышц в суставах & 28 & 28,0 & 12 & 19,05 & 40 & 24,54 \\
\hline Динамика СРБ & 16 & 16,0 & 5 & 7,94 & 21 & 12,88 \\
\hline Скованность суставов & 5 & 5,0 & 10 & 15,87 & 15 & 9,2 \\
\hline Всего & 2,94 & & 2,65 & & 2,72 & \\
\hline
\end{tabular}

Медикаментозная терапия во всех случаях включала базисные противовоспалительные препараты (БПВП): основным препаратом был метотрексат (назначен в $42 \%$ случаев). Терапия ГИБП применялась в $27 \%$ случаев, представлена в таблице 7

Не получали терапию БПВП 1 пациентов

Таблицуа 7.

Базисная терапия основного заболевания

\begin{tabular}{|l|l|l|l|}
\hline $\mathbf{n} / \mathbf{I}$ & \multicolumn{2}{|c|}{ МНН } & \multicolumn{2}{c|}{ Общее к-во случаев } \\
\hline & & Число & \% \\
\hline 1 & Тоцилизумаб & 6 & 2,21 \\
\hline 2 & Абатацепт & 19 & 7,01 \\
\hline 3 & Адалимумаб & 12 & 4,43 \\
\hline 4 & Голимумаб & 4 & 1,47 \\
\hline 5 & Лефлуномид & 14 & 5,17 \\
\hline 6 & Метилпреднизолон & 35 & 12,92 \\
\hline 7 & Метотрексат & 114 & 42,07 \\
\hline
\end{tabular}


Окончание табл. 7.

\begin{tabular}{|l|l|l|l|}
\hline 8 & Преднизолон & 2 & 0,74 \\
\hline 9 & Ритуксимаб & 8 & 2,95 \\
\hline 10 & Сульфасалазин & 1 & 0,37 \\
\hline 11 & Цертолизумаб пэгол & 24 & 8,86 \\
\hline 12 & Этанерцепт & 31 & 11,44 \\
\hline 13 & Базисную терапию не получил & 1 & 0,36 \\
\hline
\end{tabular}

\section{Обсуждение}

Установлено, что при ревматоидном артрите значительно повышен риск развития сердечно-сосудистых катастроф, в частности инфаркта миокарда. Риск развития инфаркта миокарда у больных ревматоидным артритом сопоставим с таковым у пациентов с сахарным диабетом $[1,16,17,18]$. Кроме того, у пациентов с ревматоидным артритом отмечается раннее развитие атеросклероза, тромбоза, не ишемических сердечно-сосудистых заболеваний.

На сегодняшний день становится очевидным, что традиционные принципы лечения больных ревматоидным артритом с коморбидными заболеваниями требуют пересмотра. Подход к лечению таких больных ранее подразумевал только снижение активности патологии. Однако анализ данных исследований показывает, что достижение ремиссии ревматоидного артрита не приводит к улучшению общего состояния пациентов. Кроме того, частота развития сопутствующих заболеваний может нарастать в геометрической прогрессии $[3,6]$. Поэтому ключевым моментом современной стратегии лечения ревматоидного артрита является принцип «лечим больного, а не болезнь», то есть с учетом мультиморбидного окружения. Проводимая терапия должна не только приводить к снижению активности ревматоидного артрита, но и способствовать достижению ремиссии сопутствующих заболеваний. Интерес к коморбидным состояниям при РА обусловлен их влиянием на выбор тактики лечения, эффективность и безопасность терапии, возможность достижения ремиссии, являющейся ключевым моментом современной стратегии лечения РА до достижения цели - Treat to Target (T2T) [22].

Согласно современной концепции лечения ревматоидного артрита, его надо начинать сразу после постановки диагноза, используя рекомендованные дозы синтетических БПВП $[4,22]$. Целью лечения является достижение ремиссии или низкой активности заболевания. В реальной практике не всегда возможно достигнуть данной цели. В нашем исследовании только у 40\% больных, обследованных в динамике, констатировано достижение целевых показателей. Наличие коморбидной патологии наряду с поздней диагностикой и обращаемостью за медицинской помощью является причи- 
ной низкой эффективности лечения. Наличие сопутствующих заболеваний, согласно проведенному исследованию, выявленных у $63 \%$ больных РА, создает дополнительные трудности при подборе терапии, является причиной полипрагмазии и увеличивает риск развития неблагоприятных реакций.

\section{Заключение}

У больных РА отмечена высокая частота коморбидной патологии. В структуре коморбидых состояний преобладают артериальная гипертензия и остеоартроз, а обострение хронических заболеваний желудочно-кишечного тракта и мочевыводящих путей часто затрудняет подбор базисной терапии, является причиной полипрогмазии.

\section{Список литературы}

1. Верткин А.Л., Румянцев М.А., Скотников А.С. и др. Коморбидность. Вестник семейной медицины. 2011;(3):40-7.

2. Гаспарян А.А., Меньшикова И.В., Каневская М.З. Коморбидность при ревматоидном артрите: особенности течения сердечно-сосудистых заболеваний. Клиническая медицина. 2016; 94(10). С. 745-753.

3. Гордеев А.В., Галушко Е.А., Насонов Е.Л. Концепция мультиморбидности в ревматологической практике. Научно-практическая ревматология. 2014;52(4):362-5.

4. Насонов Е.Л., Каратеев Д.Е., Чичасова Н.В. Рекомендации EULAR по лечению ревматоидного артрита - 2013: общая характеристика и дискуссионные проблемы. Научно-практическая ревматология. 2013;51 (6):609-22.

5. Насонов Е.Л. Ревматология: клинические рекомендации. 2-е изд., испр. и доп. Москва: ГЭОТАРМедиа; 2010. 752 с.

6. Никитина Н.М., Афанасьев И.А., Романова Т.А., Ребров А.П. Особенности коморбидности у больных ревматоидным артритом в разные годы наблюдения. Современная ревматология. №1’15. 39-43.

7. Панафидина Т.А., Кондратьева Л.В., Герасимова Е.В. и др. Коморбидность при ревматоидном артрите. Научно-практическая ревматология. 2014;52(3):283-289.

8. Попкова Т.В., Новикова Д.С., Насонов Е.Л. Атеротромбоз при аутоиммунных заболеваниях: современное стояние проблемы. Consilium medicum. 2008;10(11):128-35.

9. Стародубцева И.А., Васильева Л.В. Оценка переносимости и безопасности комплексной терапии вторичного остеоартроза у больных ревматоидным артритом с учетом коморбидной патологии. Медицинский совет, №5, 2016. C. $120-123$. 
10. Boers M, Dijkmans B, Gabriel S, et al. Making an impact on mortality in rheumatoid arthritis. Targeting cardiovascular comorbidity. Arthritis Rheum. 2004;50:1734-9. doi:http://dx.doi.org/10.1002/art.20306

11. Charlson ME. A new method of classifying prognostic comorbidity in longitudinal studies: development and validation. J Chron Dis. 1987;40(5):373-83. doi: 10.1016/0021-9681(87)90171-8

12. Franklin J, Lunt M, Bunn D, et al. Risk and predictors of infection leading to hospitalisation in a large primary-care-derived cohort of patients with inflammatory polyarthritis. Ann Rheum Dis. 2007;66:308-312. doi: 10.1136/ard.2006.057265

13. Gabriel SE, Michaud K. Epidemiological studies in incidence, prevalence, mortality, and comorbidity of the rheumatic diseases. Arthritis Res Ther. 2009;11(3):229. doi 10.1186/ar2669

14. Gabriel SE, Crowson CS, Kremers HM, et al. Survival in rheumatoid arthritis: a population-based analysis of trends over 40 years. Arthritis Rheum. 2003;48:54 8. doi: 10.1002/art.10705

15. Jurgens MS, Jacobs JW, Bijlsma JW. The use of conventional diseasemodifying anti-rheumatic drugs in established RA. Best Pract Res Clin Rheumatol. 2011;25(4):523-33. doi: 10.1016/j.berh.2011.10.006

16. Kadam UT, Jordan K, Croft PR. Clinical comorbidity in patients with osteoarthritis: a case-control study of general practice consulters in England and Wales. Ann Rheum Dis. 2004;63:408-10. doi: 10.1136/ard.2003.007526

17. Kitas GD, Erb N. Tackling ischemic heart disease in rheumatoid arthritis. Rheumatology. 2003;42:607-13. doi: 10.1093/rheumatology/ keg175

18. Levy L, Fautrel B, Barnetche T, Schaeverbeke T. Incidence and risk of fatal myocardial infarction and stroke events in rheumatoid arthritis patients. A systematic review of the literature. Clin Exp Rheumatol. 2008;26:673-9.

19. Maradit-Kremers H, Nicola PJ, Crowsoon CS, et al. Cardiovascular death in rheumatoid arthritis. Arthritis Rheum. 2005;52:722-32. doi: 10.1002/art.20878

20. Osiri M, Sattayasomboon Y. Prevalence and out-patient medical costs of comorbid conditions in patients with rheumatoid arthritis. Joint Bone Spine. 2013;80(6):608-12. doi:10.1016/j.jbspin.2013.01.013

21. Pasceri V, Yeh ETH. A tale of two diseases. Atherosclerosis and rheumatoid arthritis. Circulation. 1999;100:2124-6. doi: 10.1161/01.CIR.100.21.2124

22. Sattar N, McCrey DW, Capell H, McInnes IB. Explaining how «high-grade» systemic inflammation accelerated vascular risk in rheumatoid arthritis. Circulation. 2003;108:2957-63. doi: 10.1161/01.CIR.0000099844.31524.05

23. Smolen JS, Aletaha D, Bijlsma JW, et al. Treating rheumatoid.arthritis to target: recommendations of an international task force. Ann Rheum Dis. 2010;69(4):631-7. doi: 10.1136/ard.2009.123919 
24. Smolen JS, Landewe R, Breedveld FC, et al. EULAR recomendattions for the management of rheumatoid arthritis with syntethetic and biological disease-modifying antirheumatic drugs. Ann Rheum Dis. 2014;73:492-509. doi: 10.1136/annrheumdis-2013-204573

\section{References}

1. Vertkin A.L., Rumyantsev M.A., Skotnikov A.S. et al. Vestnik semeynoy meditsiny. 2011;(3):40-7.

2. Gasparyan A.A., Men'shikova I.V., Kanevskaya M.Z. Klinicheskaya meditsina. 2016; 94(10), pp. 745-753.

3. Gordeev A.V., Galushko E.A., Nasonov E.L. Nauchno-prakticheskaya revmatologiya. 2014;52(4):362-5.

4. Nasonov E.L., Karateev D.E., Chichasova N.V. Nauchno-prakticheskaya revmatologiya. 2013;51 (6):609-22

5. Nasonov E.L. Revmatologiya: klinicheskie rekomendatsii [Rheumatology: clinical recommendations]. Moskva: GEOTARMedia; 2010. 752 p.

6. Nikitina N.M., Afanas'ev I.A., Romanova T.A., Rebrov A.P. Sovremennaya revmatologiya. №1'15. P. 39-43.

7. Panafidina T.A., Kondrat'eva L.V., Gerasimova E.V. et al. Nauchno-prakticheskaya revmatologiya. 2014;52(3):283-289.

8. Popkova T.V., Novikova D.S., Nasonov E.L. Consilium medicum. 2008;10(11):128-35.

9. Starodubtseva I.A., Vasil'eva L.V. Meditsinskiy sovet, №5, 2016. P. 120-123

10. Boers M, Dijkmans B, Gabriel S, et al. Making an impact on mortality in rheumatoid arthritis. Targeting cardiovascular comorbidity. Arthritis Rheum. 2004;50:1734-9. doi: http://dx.doi.org/10.1002/art.20306

11. Charlson ME. A new method of classifying prognostic comorbidity in longitudinal studies: development and validation. J Chron Dis. 1987;40(5):373-83. doi: 10.1016/0021-9681(87)90171-8

12. Franklin J, Lunt M, Bunn D, et al. Risk and predictors of infection leading to hospitalisation in a large primary-care-derived cohort of patients with inflammatory polyarthritis. Ann Rheum Dis. 2007;66:308-312. doi: 10.1136/ard.2006.057265

13. Gabriel SE, Michaud K. Epidemiological studies in incidence, prevalence, mortality, and comorbidity of the rheumatic diseases. Arthritis Res Ther. 2009;11(3):229. doi 10.1186/ar2669

14. Gabriel SE, Crowson CS, Kremers HM, et al. Survival in rheumatoid arthritis: a population-based analysis of trends over 40 years. Arthritis Rheum. 2003;48:548. doi: 10.1002/art.10705

15. Jurgens MS, Jacobs JW, Bijlsma JW. The use of conventional diseasemodifying anti-rheumatic drugs in established RA. Best Pract Res Clin Rheumatol. 2011;25(4):523-33. doi:10.1016/j.berh.2011.10.006 
16. Kadam UT, Jordan K, Croft PR. Clinical comorbidity in patients with osteoarthritis: a case-control study of general practice consulters in England and Wales. Ann Rheum Dis. 2004;63:408-10. doi: 10.1136/ard.2003.007526

17. Kitas GD, Erb N. Tackling ischemic heart disease in rheumatoid arthritis. Rheumatology. 2003;42:607-13. doi: 10.1093/rheumatology/ keg175

18. Levy L, Fautrel B, Barnetche T, Schaeverbeke T. Incidence and risk of fatal myocardial infarction and stroke events in rheumatoid arthritis patients. A systematic review of the literature. Clin Exp Rheumatol. 2008;26:673-9.

19. Maradit-Kremers H, Nicola PJ, Crowsoon CS, et al. Cardiovascular death in rheumatoid arthritis. Arthritis Rheum. 2005;52:722-32. doi: 10.1002/art.20878

20. Osiri M, Sattayasomboon Y. Prevalence and out-patient medical costs of comorbid conditions in patients with rheumatoid arthritis. Joint Bone Spine. 2013;80(6):608-12. doi:10.1016/j.jbspin.2013.01.013

21. Pasceri V, Yeh ETH. A tale of two diseases. Atherosclerosis and rheumatoid arthritis. Circulation. 1999;100:2124-6. doi: 10.1161/01.CIR.100.21.2124

22. Sattar N, McCrey DW, Capell H, McInnes IB. Explaining how «high-grade» systemic inflammation accelerated vascular risk in rheumatoid arthritis. $\mathrm{Circu}$ lation. 2003;108:2957-63. doi: 10.1161/01.CIR.0000099844.31524.05

23. Smolen JS, Aletaha D, Bijlsma JW, et al. Treating rheumatoid.arthritis to target: recommendations of an international task force. Ann Rheum Dis. 2010;69(4):631-7. doi: 10.1136/ard.2009.123919

24. Smolen JS, Landewe R, Breedveld FC, et al. EULAR recomendattions for the management of rheumatoid arthritis with syntethetic and biological disease-modifying antirheumatic drugs. Ann Rheum Dis. 2014;73:492-509. doi: 10.1136/annrheumdis-2013-204573

\section{ДАННЫЕ ОБ АВТОРАХ}

Хусаинова Д.И., Бакиров Б.А., Зарипова Г.Р., Хусаинова Л.Н., Аитова Э.М., Баянова И.Л., Музаева И.Л., Хакамова Г.А.

Башкирский государственный медииинский университет ул. Ленина, 3, Уфа, Респ. Башкортостан, 450008, Российская Федераиия dianochka.xusainova@mail.ru

\section{DATA ABOUT THE AUTHORS}

Khusainova D.I., Bakirov B.A., Zaripova G.R., Khusainova L.N., Aitova E.M., Bayanova I.L., Muzaeva I.L., Khakamova G.A. Bashkir State Medical University

3, Lenin Str., Ufa, Bashkortostan, 450008, Russian Federation dianochka.xusainova@mail.ru 